\title{
ON A CONSTRUCTION OF BREDON
}

\author{
JOSEPH ROITBER.G ${ }^{1}$
}

\begin{abstract}
Using a homotopy-theoretical description of a geometric pairing due to Bredon, we show how to rederive Bredon's results on the pairing. Furthermore, we are able to, in some sense, complete these results by combining the homotopy-theoretical approach with Sullivan's determination of the 2-primary Postnikov decomposition of the space $G / P L$.
\end{abstract}

1. Introduction. In [1], Bredon introduced a geometric pairing

$$
\Gamma_{n} \times \Pi_{n+k}\left(S^{n}\right) \stackrel{\rho_{n, k}}{\longrightarrow} \Gamma_{n+k},
$$

$\Gamma_{i}$ being the group of differential structures on the $i$-sphere ${ }^{2}$ and used this pairing to construct certain semifree actions of groups on spheres. There is also the pairing

$$
\Pi_{n}(P L / O) \times \Pi_{n+k}\left(S^{n}\right) \stackrel{\gamma_{n . k}}{\longrightarrow} \Pi_{n+k}(P L / O)
$$

defined by composition, $P L / O$ being the fibre of the natural map $B O \rightarrow$ $B P L$ of classifying spaces. Since, by smoothing theory, $\Pi_{n}(P L / O)$ and $\Gamma_{n}$ are isomorphic groups, it is natural to inquire about the relation between these two pairings. In fact, as observed by Bredon [1] and the present author [3], these two pairings coincide.

While Bredon's geometric definition is rather natural and simple, the main results and proofs are more perspicuous using the homotopytheoretical definition. Thus, in $\$ 2$ we show how to retrieve (and slightly generalize) the theorems of $[\mathbf{1}, \xi \S 1,2]$ in this context, in particular the following important result, which we state as

Theorem A [1, Theorem 2.1]. Let $\alpha \in \Pi_{n+k}\left(S^{n}\right), \sigma \in \Gamma_{n}$ and let $p_{l}(\sigma) \subset \Pi_{n+l}\left(S^{l}\right)$ denote the set of all elements represented (via the Pontryagin-Thom construction) by framed embeddings $\Sigma^{n} \times D^{l} \subset S^{n+l}, \Sigma^{n}$

Received by the editors September 15, 1971.

AMS 1970 subject classifications. Primary 57D55.

Key words and phrases. Bredon pairing, differential structures on spheres, $P L / O$, Kervaire-Milnor construction $p_{l}(\sigma)$, homotopy spheres bounding $\pi$-manifolds, $G / P L$.

${ }^{1}$ Partially supported by NSF grant.

2 Actually, Bredon defines his map from $\Theta_{n} \times \Pi_{n+k}\left(S^{n}\right)$ to $\Theta_{n+k}$, but it is easily seen that this map restricts to a map from $\Gamma_{n} \times \Pi_{n+k}\left(S^{n}\right)$ to $\Gamma_{n+k}$.

(c) American Mathematical Society 1972 
a homotopy sphere representing $\sigma$. Then

$$
p_{l}(\sigma) \circ \Sigma^{l} \alpha \subset p_{l}\left(\rho_{n, k}(\sigma, \alpha)\right),
$$

where $\Sigma^{i} \alpha$ is the lth iterated suspension of $\alpha$.

However, the main contribution of this note is the following Theorem B (essentially conjectured by Bredon [1, Corollary 2.3 and succeeding remarks]) which, taken together with Theorem A, gives a rather good hold on Bredon's pairing.

THEOREM B. If $b P_{n+1}$ is the subset of $\Gamma_{n}$ consisting of those homotopy spheres which bound $\Pi$-manifolds, then the pairing $\rho_{n, k}$ restricts to a pairing $\bar{\rho}_{n, k}: b P_{n+1} \times \Pi_{n+k}\left(S^{n}\right) \rightarrow b P_{n+k+1}$. Moreover, $\bar{\rho}_{n, k}$ is trivial for $k>0$.

The proof of Theorem B, which relies on results of Sullivan [4], will also be carried out in $\S 2$.

As a final introductory comment, we remark that while the main interest in the composition pairing $\Pi_{n}(P L / O) \times \Pi_{n+k}\left(S^{n}\right) \rightarrow \Pi_{n+k}(P L / O)$ lies in its geometric interpretation, the pairing has also proved to be of some use in studying the $k$-invariants of $P L / O$ ([5], [3]).

2. The main properties of $\rho_{n, k}$. In this section we deduce the main properties of the pairing $\rho_{n, k}$, working in the homotopy-theoretical context. We begin with a proposition which summarizes and slightly generalizes the results of $[1, \S 1]$.

PROPOSITION. The pairing $\rho_{n, k}: \Gamma_{n} \times \Pi_{n+k}\left(S^{n}\right) \rightarrow \Gamma_{n+k}$ is bilinear and associative in the sense that the diagram

$$
\begin{aligned}
& \Gamma_{n} \times \Pi_{n+k}\left(S^{n}\right) \times \Pi_{n+k+l}\left(S^{n+k}\right) \stackrel{\mathrm{id} \times \text { comp }}{\longrightarrow} \Gamma_{n} \times \Pi_{n+k+l}\left(S^{n}\right) \\
& \downarrow^{\rho_{n, k}} \times \text { id } \quad \rho^{\rho_{n, k+l}} \\
& \Gamma_{n+k} \times \Pi_{n+k+l}\left(S^{n+k}\right) \longrightarrow \rho_{n+k . l} \longrightarrow \Gamma_{n+k+l}
\end{aligned}
$$

is commutative.

Proof. Using the identification of $\rho_{n, k}$ with $\gamma_{n, k}$, all these statements, except perhaps the linearity in the first variable, are trivial. As for linearity in the first variable, this follows from the fact that $P L / O$ is an $H$-space. ${ }^{3}$

We remark that linearity in the first variable was proved in [1] only under the assumption that $\Pi_{n+k}\left(S^{n}\right)$ is stable, i.e. $k<n-1$. Our proof, exploiting the $H$-structure on $P L / O$, shows this restriction on $k$ to be unnecessary.

\footnotetext{
${ }^{3}$ The bilinearity of $\gamma_{n, k}$ has been independently observed by Schultz (Smooth structures on $S^{p} \times S^{q}$, Ann. of Math. (2) 90 (1969), 187-198) in a closely related context.
} 
We come now to the two key results, Theorems A and B.

Proof of TheOREM A. Let $\Gamma_{n+l, n}^{f}$ denote the set of framed embeddings of homotopy $n$-spheres in $S^{n+l}, G_{l}^{+}$the space of maps $S^{l-1} \rightarrow S^{l-1}$ of degree 1. In addition to the obvious (forgetful) map $q_{i} \cdot \Gamma_{i+l . i}^{f} \rightarrow \Gamma_{i}$, there is a map $\omega_{i}: \Gamma_{i+l, i}^{f} \rightarrow \Pi_{i}\left(G_{l}^{+}\right)$described in [2]. ${ }^{4}$ It is not difficult to see that the set $p_{l}(\sigma)$, as originally defined by Kervaire-Milnor, can equivalently be described as $J_{n}\left(\omega_{n}\left(\varphi_{n}^{-1}(\sigma)\right)\right)$, where $J_{n}: \Pi_{n}\left(G_{l}^{+}\right) \rightarrow \Pi_{n+l}\left(S^{l}\right)$ is obtained, as usual, by the Hopf construction. Thus, if $\beta \in p_{l}(\sigma)$, there exists $\eta \in$ $\omega_{n}\left(\varphi_{n}^{-1}(\sigma)\right)$ such that $\beta=J_{n}(\eta)$. But by a known formula (cf. [1, p. 442]) we have $J_{n}(\eta) \circ \Sigma^{l} \alpha=J_{n+k}(\eta \circ \alpha)$. Moreover, it is not difficult to see, for example by using the homotopy-theoretical interpretation of $\Gamma_{i+l, i}^{f}, \varphi_{i}, \omega_{i}$ (see footnote 4) that $\eta \circ x \in \omega_{n+k}\left(\varphi_{n+k}^{-1}\left(\rho_{n, k}(\sigma, \alpha)\right)\right.$ and the theorem follows.

Proof of THEOREM B. If $G_{k}$ is the set of maps $S^{k-1} \rightarrow S^{k-1}$ of degree \pm 1 and $G=\lim _{k \rightarrow \infty} G_{k}$, there are natural maps $O \subset P L \subset G$ and a fibration $P L / O \rightarrow G / O \rightarrow G / P L$. For $\alpha \in \Pi_{n+k}\left(S^{n}\right)$, we consider the diagram.

$$
\begin{gathered}
\Pi_{n+1}(G / P L) \stackrel{\partial_{n}}{\longrightarrow} \Pi_{n}(P L / O) \\
\downarrow \\
\Pi_{n+k+1}(G / P L) \stackrel{\partial_{n+k}}{\longrightarrow} \Pi_{n+k}(P L / O)
\end{gathered}
$$

The commutativity of (2.1) is a consequence of a well-known formula in fibre-space theory. From [4], we know that $b P_{i+1}$ can be described homotopy-theoretically as the image of $\partial_{i}: \Pi_{i+1}(G / P L) \rightarrow \Pi_{i}(P L / O)$, so to prove the theorem, it is sufficient to prove the left-hand vertical arrow in (2.1) is the zero map. ${ }^{5}$

To this end, observe first that since $k>0, \Sigma \alpha$ has finite order. We may further clearly assume $\Sigma \propto$ has prime-power order $p^{j}$ and distinguish two cases, according as $p$ is odd or $p=2$.

For $p$ odd, the conciusion is trivial because the only torsion in $\Pi_{*}(G / P L)$ is of order 2 [4]. If $p=2$, we argue as follows. Let $Y$ be the space

$$
\left(K\left(Z_{2}, 2\right) \times_{\delta S q^{2}} K(Z, 4)\right) \times \Pi_{i \geqq 2} K\left(Z_{2}, 4 i-2\right) \times K(Z, 4 i) .
$$

Sullivan [4] shows that after localizing at the prime 2, $Y$ and $G / P L$ become homotopy equivalent. We are therefore reduced to proving that $\Pi_{n+1}(Y)^{\circ \Sigma_{\alpha}} \Pi_{n+k+1}(Y)$ is the zero map, which is evident from the structure of $Y$.

\footnotetext{
${ }^{4}$ See Rourke-Sandersor, Block bundles. III, Ann. of Math. (2) 87 (1968), 431-483, for a homotopy-theoretic description of the set $\Gamma_{i+l, i}^{j}$ and the maps $\varphi_{i}, \omega_{i}$.

${ }^{5}$ It is actually true that the composition pairing $\Pi_{n+1}(G / P L) \times \Pi_{n+k+1}\left(S^{n+1}\right) \rightarrow$ $\Pi_{n+k+1}(G / P L)$ is trivial but we do not need this additional fact.
} 


\section{BIBLIOGRAPHY}

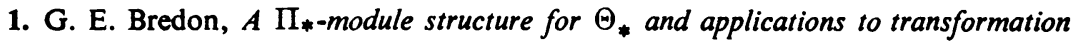
groups, Ann. of Math. (2) 86 (1967), 434-448. MR 36 \#4570.

2. J. Levine, A classification of differentiable knots, Ann. of Math. (2) 82 (1965), 15-50. MR 31 \#5211.

3. J. Roitberg, PL invariants on a smooth manifold, Thesis, New York University, New York, 1968.

4. D. Sullivan, Triangulating homotopy equivalences, Thesis, Princeton University, Princeton, N.J., 1965.

5. R. Williamson, Jr., Cobordism of combinatorial manifolds, Ann. of Math. (2) 83 (1966), 1-33. MR 32 \#1715.

Department of Mathematics, State University of New York at Stony Brook, STONY BROOK, NEW YORK 11790 